\title{
Measurements of human vision contrast sensitivity to opposite colors using a cathode ray tube display
}

\author{
YAO JunCai \\ Department of Physics, Shaanxi University of Technology, Hanzhong 723000, China
}

Received March 22, 2011; accepted May 16, 2011

\begin{abstract}
The contrast definitions of opposite colors red-green and blue-yellow, which have been described in the CIELAB and $\mathrm{YC}_{\mathrm{r}} \mathrm{C}_{\mathrm{b}}$ color spaces, respectively, and a method to accurately control chroma and color differences on a cathode ray tube screen are presented according to the color display characteristics of cathode ray tubes. Using target gratings on a cathode ray tube screen, measurements of human vision contrast sensitivity to red-green and blue-yellow colors for 10 spatial frequencies $(0.41,0.82,1.23,1.97$, $3.08,3.79,4.93,7.04,9.86$ and $16.43 \mathrm{cpd}$ ) were carried out in 11 young observers in a dark environment. Contrast sensitivity functions of opposite colors were obtained, which can be used for practical applications in image technologies.
\end{abstract}

color vision, contrast sensitivity, target grating, cathode ray tube display

Citation: Yao J C. Measurements of human vision contrast sensitivity to opposite colors using a cathode ray tube display. Chinese Sci Bull, 2011, 56: 2425-2432, doi: $10.1007 / \mathrm{s} 11434-011-4595-8$

The contrast sensitivity function (CSF) is central to describing spatial vision and to models of visual coding. The CSF describes the relationship between spatial frequencies and contrast sensitivity which is described by the reciprocal of the contrast detection threshold. Measurements of visual contrast sensitivity are fundamental to modeling human vision and predicting visual performance. Psychophysical experiments can be influenced by many factors, such as instrument performance, precision of the metering equipment and the experimental environment and technique [1-7]. Some researchers are concerned with both studying the visual system and developing image technology. Early research about CSFs has mainly concentrated on the luminance contrast sensitivity. Research studies and applications of human vision color transmission characteristics have only begun recently. However, color CSFs have greater application value than luminance CSFs in electro-optical image technology. With the development of the computer, networks and digital technologies, the cathode ray tube (CRT) display has become the primary means of presenting

email: yjc4782334@yahoo.com.cn information, and image technology for the displays have become more comprehensive. In this regard, measurements of color CSFs on a CRT display have increasing importance and are of significant practical application value [8-10].

In 2001, Wenzel studied the differences in contrast sensitivity between normal and defective human vision using a CRT display [5]. In 2000, Nadenau measured contrast sensitivity of human vision to luminance and red-green and blue-yellow colors, in CIELAB and $\mathrm{YC}_{\mathrm{r}} \mathrm{C}_{\mathrm{b}}$ color spaces, respectively, to study the image compression algorithm [7]. In 2004, Owens et al. measured the contrast sensitivity of human vision to various single colors; i.e. red, green, blue and yellow, which were displayed on a colored background, using a CRT monitor [3]. However, they did not provide the details of the measuring conditions, measuring process, target gratings and color contrast definitions. In this study, contrast definitions of red-green and blue-yellow color stripes are proposed for CIELAB and $\mathrm{YC}_{\mathrm{r}} \mathrm{C}_{\mathrm{b}}$ color spaces, respectively, according to the experiences of previous studies and color display characteristics of a CRT [11-24]. A method is proposed to accurately control the color of a CRT display in a small range and a method for measuring 
contrast sensitivity to red-green and blue-yellow opposite colors on a CRT display are detailed. Measurements were carried out for 10 spatial frequencies $(0.41,0.82,1.23,1.97$, $3.08,3.79,4.93,7.04,9.86$ and $16.43 \mathrm{cpd}$ ) in 11 young observers, and CSFs of the opposite colors red-green and blue-yellow under dark adapted conditions were obtained.

\section{Methods}

\subsection{Contrast definitions for red-green and blue-yellow colors}

Human vision is based on differentiating and recognizing environmental surface parts with different colors and luminances that are based on variations in light reaching the retina. The differentiation of surface parts with different luminance and color is based on luminance and color contrast. If there are different luminance areas and colors in the visual field, the contrast between the images is apparent. Therefore, two aspects, luminance and color, must be considered simultaneously when measuring an image's color contrast [3-5]. For numerical evaluation to be possible, the red-green and blue-yellow color contrast definitions are interpreted as follows.

Because the CIELAB color space does not vary with equipment variation, in color management, the original color, screen color and printing color in the color space may be calculated and communicated according to the corresponding relationships to achieve color coincidence in the vision and the realization of color conversion parameters for different equipment $[10,11]$. The color space of $\mathrm{YC}_{\mathrm{r}} \mathrm{C}_{\mathrm{b}}$ for television signals is used in the international BT.601 digital television signal, which is obtained by zooming and translating from the color space of YUV [10,11]. If measurements are carried out on a CRT display to obtain a CSF, the color space of $\mathrm{YC}_{\mathrm{r}} \mathrm{C}_{\mathrm{b}}$ is very suitable.

According to the above considerations, the contrast definitions of red-green and blue-yellow color stripes are proposed in this work, which are based on the color differences and the color systems of CIELAB and $\mathrm{YC}_{\mathrm{r}} \mathrm{C}_{\mathrm{b}}$ color spaces. The definitions were determined as follows. Some color spot in the color space was chosen, whose chroma is recorded as $a^{*}=0, b^{*}=0$ or $C_{r}=0, C_{b}=0$, which are the values of the coordinate origin in the reference frame of Figures 1 and 2. $-a^{*}$ or $-C_{r}$ means that the color is leaning toward green, $+a^{*}$ or $+C_{r}$ means that the color is leaning toward red. $+b^{*}$ or $-C_{b}$ means that the color is leaning toward yellow, and $-b^{*}$ or $+C_{b}$ means that the color is leaning toward blue. The target gratings were composed of red-green or blue-yellow color stripes. $\bar{Y}$ is described as the mean luminance of the gratings, which is kept constant and whose unit is $\mathrm{cd} / \mathrm{m}^{2}$. The value of $b^{*}$ or $C_{b}$ is zero when measuring the contrast sensitivity to the red-green color. And the value of $a^{*}$ or $C_{r}$ is zero when measuring the contrast sensitivity to the blue-yellow color. According to the color difference definition of the CIE $1976 L^{*} a^{*} b^{*}$ even color space, the contrast definition of opposite color stripes in CIELAB and $\mathrm{YC}_{\mathrm{r}} \mathrm{C}_{\mathrm{b}}$ color spaces are indicated as eqs. (1) and (2), respectively. Thus, $a_{1}^{*}, b_{1}^{*}, C_{r 1}, C_{b 1}$ and $a_{2}^{*}, b_{2}^{*}, C_{r 2}, C_{b 2}$ can be described as the chroma of two opposite color blocks.

$$
\begin{gathered}
C_{a}=\frac{\Delta a^{*}}{\bar{Y}}, \quad C_{b}=\frac{\Delta b^{*}}{\bar{Y}}, \Delta a^{*}=\left|a_{2}^{*}-a_{1}^{*}\right|, \quad \Delta b^{*}=\left|b_{2}^{*}-b_{1}^{*}\right|, \\
C_{C_{r}}=\frac{\Delta C_{r}}{\bar{Y}}, C_{C_{b}}=\frac{\Delta C_{b}}{\bar{Y}}, \\
\Delta C_{r}=\left|C_{r 2}-C_{r 1}\right|, \Delta C_{b}=\left|C_{b 2}-C_{b 1}\right| .
\end{gathered}
$$

\subsection{Experimental instruments and parameters}

We used a Sony-G520, 21 inch CRT that had been used for 2 years, approximately for $4500 \mathrm{~h}$, with a displaying effect equivalent to a new monitor. The image gathering

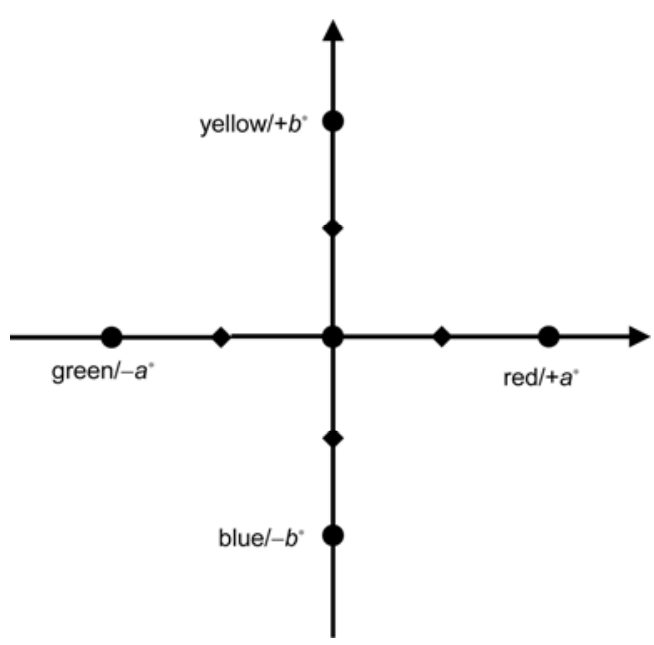

Figure 1 Founding color coordinate reference with origin of $a^{*}=b^{*}=0$ selected in CIELAB color space.

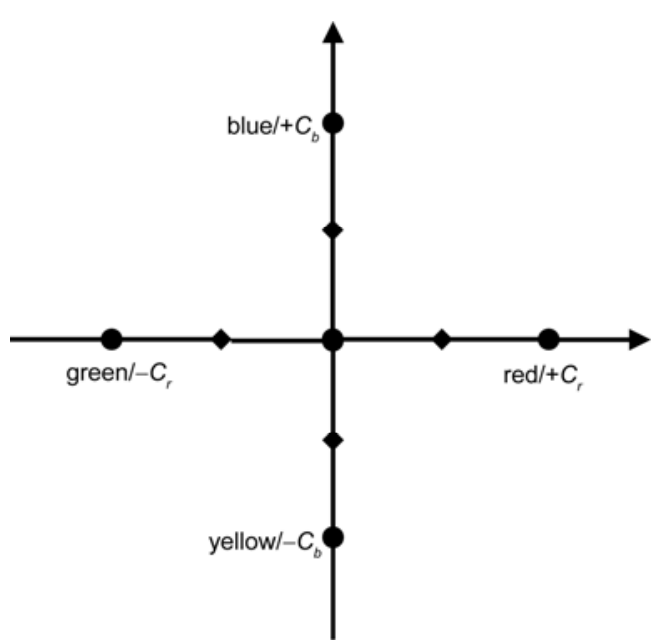

Figrue 2 Founding color coordinate reference with origin of $C_{r}=C_{b}=0$ selected in $\mathrm{YC}_{\mathrm{r}} \mathrm{C}_{\mathrm{b}}$ color space. 
card was a Matrox Millennium P650 real-time image gathering card with 10 bit-digital high definition and a stable color display. The screen chronometer was made by the American X-Rite Corporation. The screen photometer was a ST-86LA model with a measuring luminance range between 0.01-19990 cd/ $/ \mathrm{m}^{2}$, made by the Electro-optic Instruments Factory of Beijing Normal University.

Target gratings with 10 spatial frequencies $(0.41,0.82$, $1.23,1.97,3.08,3.79,4.93,7.04,9.86$ and $16.43 \mathrm{cpd})$ were vertical rectangle stripes, with a mean luminance of 52.5 $\mathrm{cd} / \mathrm{m}^{2}$ or $54.5 \mathrm{~cd} / \mathrm{m}^{2}$. The color block was $8.5 \mathrm{~cm} \times 8.5 \mathrm{~cm}$ in size, located in the center of the screen of the display, with a grey background that was $27.6 \mathrm{~cd} / \mathrm{m}^{2}$ in luminance. The spatial viewing angle was $2.4347^{\circ}$ and the viewing distance was $2 \mathrm{~m}$. The CRT display was set into white $\mathrm{D}_{65}$ (measured real values: $x=0.3122, y=0.3292, Y=100.36$ ), with the greatest luminance at $100.28 \mathrm{~cd} / \mathrm{m}^{2}$. Measurements were carried out under dark adapted conditions with standard grey walls. Observers were 11 young (20-32-years-old) people with normal vision, who were undergraduate or graduate students.

\subsection{Method for precise control of chroma $\left(L, a^{*}, b^{*}\right)$ and color differences on a CRT in a small range}

Measurements of human vision contrast sensitivity are psychophysical tasks, which are influenced by many factors [1-7]. For the measurement of contrast sensitivity using a CRT display, the most important factor is how to control the chroma and color differences on the CRT display. The contrast of target gratings of red-green and blue-yellow colors must be controlled accurately with the three channels of the CRT in coordination. To accurately control the chroma and color differences, a CRT display must recognize the following four requests. The first is that requests for the contrast of opposite color stripes, which are defined as color differences in CIELAB, must be satisfied. Namely, when values of $a^{*}$ or $b^{*}$ change, it must be guaranteed that corresponding values of $b^{*}$ or $a^{*}$ are zero or approaching zero ( $C_{r}$ or $C_{b}$ requirements are similar). The second is that the display must be accurately controlled in the medium term while measurements are being completed. The third is that the contrast values of gratings that the display card can realize must be lower than contrast detection thresholds of human vision. The last is how to manufacture target gratings that accurately display color on the CRT. A method is proposed to solve the above problems, which is as follows.

The color on the CRT display follows the color principles of the RGB color space, and relies on the particular equipment. Because the three channels of a CRT suppress mutually, the relationship between the RGB color space and the CIEXYZ color space which does not vary with variation in the equipment, must be established to realize an open style of color management. It is then transformed into the CIELAB color space again $[12,13]$.
To date, the application of the lookup table (LUT) method or GOG model to characterize a CRT display generally involves two steps. The first step is a non-linear process that forms the luminance of the electron guns, resulting from a digital quantity displayed by three channels of the display. The second step is to obtain CIEXYZ chromaticity through the luminance of three electron guns. Previous experiments demonstrated that the first step does not directly take into account the alternating effect among the three channels, but indirectly involves it when measuring the luminance of a neutral grey in the non-linear process of obtaining the luminance from electron guns by a digital quantity driving the three channels of the display. If the second step is used directly without the first step, the requirement for greater precision in the control of the CRT display color in a small range cannot be fulfilled (the precision for characterizing a CRT with the LUT method and GOG model are $0.42 \Delta E^{*} a b$ and $0.54 \Delta E^{*} a b$, respectively). Because a conventional computer adopts an 8 bit image display card using three channels, the requests for grating contrasts that the display can realize must be lower than the contrast detection thresholds of human vision. In this experiment, a 10 bit image display card using three channels was adopted. Namely, three values $(0.25,0.50$ and 0.75$)$ were inserted into the chroma values of RGB, or R, or $\mathrm{G}$ or $\mathrm{B}$ of the 8 bit display whose gap is 1 , to describe color to match the requests. According to the gamma characteristic of luminance and color display characteristic of the CRT, on the basis of linear interpolation, a method is put forward to accurately control the chroma and color differences of the CRT display in a small range, which takes into account directly interaction among the three channels and instability of the display in the medium term, to realize the conversion among arbitrary combination values of $\mathrm{R}, \mathrm{G}$ and $\mathrm{B}$ in the CRT color space, CIEXYZ and CIELAB chromaticity values. This allowed us to meet the demands for accurately controlling and realizing the smaller contrast values of target gratings. The exact method is described as follows.

Step 1: Initially characterize the CRT display with the LUT method; choose the values of R, G and B in a small color range of the CRT display and the interval of 1 between two values; change the values of each channel, and then measure the tri-stimulus values of $X_{\mathrm{R}}, Y_{\mathrm{R}}, Z_{\mathrm{R}}, X_{\mathrm{G}}, Y_{\mathrm{G}}$, $Z_{\mathrm{G}}, X_{\mathrm{B}}, Y_{\mathrm{B}}$ and $Z_{\mathrm{B}}$ when three channels are working separately.

Step 2: Measure tri-stimulus values of $X_{\mathrm{R}=\mathrm{G}=\mathrm{B}}, Y_{\mathrm{R}=\mathrm{G}=\mathrm{B}}$ and $Z_{\mathrm{R}=\mathrm{G}=\mathrm{B}}$ when three channels are working together, with an interval of 1 between two values and changing values RGB $(\mathrm{R}=\mathrm{G}=\mathrm{B})$ in a small range.

Step 3: (a) Work out the differences between the tri-stimulus values of $X_{\mathrm{R}=\mathrm{G}=\mathrm{B}}, \quad Y_{\mathrm{R}=\mathrm{G}=\mathrm{B}}$ and $Z_{\mathrm{R}=\mathrm{G}=\mathrm{B}}$ and of $X_{\mathrm{R}}+X_{\mathrm{G}}+X_{\mathrm{B}}, Y_{\mathrm{R}}+Y_{\mathrm{G}}+Y_{\mathrm{B}}, Z_{\mathrm{R}}+Z_{\mathrm{G}}+Z_{\mathrm{B}}$ (see eq. (3)). (b) Based on the values above, work out the overall ratios between the tri-stimulus values presented by three guns separately and ones together, namely $x_{\mathrm{r}}=X_{\mathrm{R}} / X_{\mathrm{R}=\mathrm{G}=\mathrm{B}}, \quad x_{\mathrm{g}}=X_{\mathrm{G}} / X_{\mathrm{R}=\mathrm{G}=\mathrm{B}}$, 
$x_{\mathrm{b}}=X_{\mathrm{B}} / X_{\mathrm{R}=\mathrm{G}=\mathrm{B}}, \quad y_{\mathrm{r}}=Y_{\mathrm{R}} / Y_{\mathrm{R}=\mathrm{G}=\mathrm{B}}, \quad y_{\mathrm{g}}=Y_{\mathrm{G}} / Y_{\mathrm{R}=\mathrm{G}=\mathrm{B}}, \quad y_{\mathrm{b}}=Y_{\mathrm{B}} / Y_{\mathrm{R}=\mathrm{G}=\mathrm{B}}$, $z_{\mathrm{r}}=Z_{\mathrm{R}} / Z_{\mathrm{R}=\mathrm{G}=\mathrm{B}}, \quad z_{\mathrm{g}}=Z_{\mathrm{G}} / Z_{\mathrm{R}=\mathrm{G}=\mathrm{B}}, z_{\mathrm{b}}=Z_{\mathrm{B}} / Z_{\mathrm{R}=\mathrm{G}=\mathrm{B}}$, and solve their respective averages. (c) Add the differences to the tri-stimulus values of $X_{\mathrm{R}}, Y_{\mathrm{R}}, Z_{\mathrm{R}}, X_{\mathrm{G}}, Y_{\mathrm{G}}, Z_{\mathrm{G}}, X_{\mathrm{B}}, Y_{\mathrm{B}}$, and $Z_{\mathrm{B}}$ when presented separately, on the basis of the proportions between the tri-stimulus values presented separately by three guns and ones together (see eq. (4)). Then solved values are taken as the tri-stimulus values of $X_{\mathrm{R} 1}, Y_{\mathrm{R} 1}, Z_{\mathrm{R} 1}, X_{\mathrm{G} 1}$, $Y_{\mathrm{G} 1}, Z_{\mathrm{G} 1}, X_{\mathrm{B} 1}, Y_{\mathrm{B} 1}, Z_{\mathrm{B} 1}$ presented separately by three channels.

$$
\begin{aligned}
& {\left[\begin{array}{c}
X_{\mathrm{dif}} \\
Y_{\mathrm{dif}} \\
Z_{\mathrm{dif}}
\end{array}\right]=\left[\begin{array}{c}
X_{\mathrm{R}=\mathrm{G}=\mathrm{B}} \\
Y_{\mathrm{R}=\mathrm{G}=\mathrm{B}} \\
Z_{\mathrm{R}=\mathrm{G}=\mathrm{B}}
\end{array}\right]-\left[\begin{array}{c}
X_{\mathrm{R}}+X_{\mathrm{G}}+X_{\mathrm{B}} \\
Y_{\mathrm{R}}+Y_{\mathrm{G}}+Y_{\mathrm{B}} \\
Z_{\mathrm{R}}+Z_{\mathrm{G}}+Z_{\mathrm{B}}
\end{array}\right],} \\
& {\left[\begin{array}{ccc}
X_{\mathrm{dif}} & 0 & 0 \\
0 & Y_{\mathrm{dif}} & 0 \\
0 & 0 & Z_{\mathrm{dif}}
\end{array}\right] \cdot\left[\begin{array}{ccc}
x_{\mathrm{r}} & x_{\mathrm{g}} & x_{\mathrm{b}} \\
y_{\mathrm{r}} & y_{\mathrm{g}} & y_{\mathrm{b}} \\
z_{\mathrm{r}} & z_{\mathrm{g}} & z_{\mathrm{b}}
\end{array}\right]+\left[\begin{array}{ccc}
X_{\mathrm{R}} & X_{\mathrm{G}} & X_{\mathrm{B}} \\
Y_{\mathrm{R}} & Y_{\mathrm{G}} & Y_{\mathrm{B}} \\
Z_{\mathrm{R}} & Z_{\mathrm{G}} & Z_{\mathrm{B}}
\end{array}\right]} \\
& =\left[\begin{array}{ccc}
X_{\mathrm{R} 1} & X_{\mathrm{G} 1} & X_{\mathrm{B} 1} \\
Y_{\mathrm{R} 1} & Y_{\mathrm{G} 1} & Y_{\mathrm{B} 1} \\
Z_{\mathrm{R} 1} & Z_{\mathrm{G} 1} & Z_{\mathrm{B} 1}
\end{array}\right] .
\end{aligned}
$$

Step 4: According to the tri-stimulus values of $X_{\mathrm{R} 1}, Y_{\mathrm{R} 1}$, $Z_{\mathrm{R} 1}, X_{\mathrm{G} 1}, Y_{\mathrm{G} 1}, Z_{\mathrm{G} 1}, X_{\mathrm{B} 1}, Y_{\mathrm{B} 1}, Z_{\mathrm{B} 1}$ and the 10 bit-display card of the CRT display in the experiments, the values of $R, G$ and $\mathrm{B}$ are determined by the linear interpolation using the interval of 0.25 to establish an interpolation table for the channel (namely, a color LUT). For example, Table 1 shows the LUT of the R channel.

Step 5: According to the color addition principle, arbitrarily arrange three values of three single channels to build a LUT between the values of three single channels and the value of CIEXYZ, then converted to the CIELAB color space according to eq. (5).

If $Y / Y_{n} \geqslant 0.008856$, or $X / X_{n} \geqslant 0.008856$, or $Z / Z_{n}$ $\geqslant 0.008856$

$$
\begin{aligned}
& L=116\left(Y / Y_{n}\right)^{\frac{1}{3}}-16, a^{*}=500\left[\left(X / X_{n}\right)^{\frac{1}{3}}-\left(Y / Y_{n}\right)^{\frac{1}{3}}\right], \\
& b^{*}=200\left[\left(Y / Y_{n}\right)^{\frac{1}{3}}-\left(Z / Z_{n}\right)^{\frac{1}{3}}\right] .
\end{aligned}
$$

Thereinto $X_{n}=95.04, Y_{n}=100.0, Z_{n}=108.89$.

Else if $Y / Y_{n} \leqslant 0.008856$, or $X / X_{n} \leqslant 0.008856$, or $Z / Z_{n} \leqslant 0.008856$,

$$
\begin{aligned}
& L=903.3\left(Y / Y_{n}\right), a^{*}=3893.5\left[\left(X / X_{n}\right)-\left(Y / Y_{n}\right)\right], \\
& b^{*}=1557.4\left[\left(Y / Y_{n}\right)-\left(Z / Z_{n}\right)\right] .
\end{aligned}
$$

According to the above proposed method, an experiment was designed to test whether the method was able to realize accurate control of the chromaticity and color differences on the CRT display. The experimental method was as follows. The values of some small range of RGB and R, G and B in the display color range were arbitrarily selected. Accordingto above proposed method, the values of $X, Y$ and $Z$ corre-
Table 1 LUT of R channel

\begin{tabular}{cc}
\hline $\mathrm{R}$ & $X_{\mathrm{R}} \quad Y_{\mathrm{R}} Z_{\mathrm{R}}$ \\
\hline$\ldots \ldots$ & $\ldots \ldots$ \\
$\mathrm{R}_{0}-0.5$ & $\ldots \ldots$ \\
$\mathrm{R}_{0}-0.25$ & $\ldots \ldots$ \\
$\mathrm{R}_{0}$ & $\ldots \ldots$ \\
$\mathrm{R}_{0}+0.25$ & $\ldots \ldots$ \\
$\mathrm{R}_{0}+0.5$ & $\ldots \ldots$ \\
$\ldots \ldots$ & \\
\hline
\end{tabular}

sponding to the different combined R, G and B values, were determined, and then they were transformed into $L^{*}, a^{*}$ and $b^{*}$ values of the CIELAB color space by computation. The results of comparing the values of $L^{*}, a^{*}$ and $b^{*}$ of the CIELAB color space by computation with values from measurements in the medium term, according to the demands of measurement contrast detection thresholds, show that the control precise of the chromaticity of $Y, L^{*}, a^{*}$ and $b^{*}$ is $0.1711,0.2782,0.1108$ and 0.1134 , respectively, and the precision of the control of color differences is $0.3543 \Delta E^{*} a b$, which is more precise than the existing LUT method and the GOG model.

\subsection{Method for manufacturing target gratings}

Because contrast values of the gratings cannot be continuously changed, pictures with different contrasts that are near the human contrast detection threshold corresponding to a spatial frequency of the opposite color stripes must be finished in advance if the contrast detection threshold of some luminance and spatial frequency is to be measured.

(i) Method for manufacturing target gratings with the contrast defined in the CIELAB color space. According to the method of accurate control chromaticity and color differences on a CRT display in a small range, the mutual transformation values can be obtained among random combination values of $R, G$ and $B$ of the RGB color space on the CRT display, chromaticity values of CIEXYZ and ones of CIELAB. Based on the precision of the display, values of RGB and $X Y Z$ are selected corresponding to the values of the CIELAB color space that $\pm a^{*}$ changes from \pm 4 to zero spaced in 0.25 intervals when $b^{*}$ is zero and that $\pm b^{*}$ changes from \pm 4 to zero in 0.25 intervals when $a^{*}$ is zero. The mean luminance of the stripes of every grating is maintained at a constant luminance of $52.50 \mathrm{~cd} / \mathrm{m}^{2}$ or $54.5 \mathrm{~cd} / \mathrm{m}^{2}$. According to the contrast definition of eq. (1), the contrast values in the experiment are determined by the values of the CIELAB color space. Large numbers of pictures with different spatial frequencies and different contrast values are designed according to corresponding values of $R, G$ and $B$ that are selected and combined. The spatial frequencies of pictures are realized by adjusting the width of the stripes. The smallest contrast value of pictures may be 0.0095 .

(ii) Method for manufacturing target gratings with the 
contrast defined in the $\mathrm{YC}_{\mathrm{r}} \mathrm{C}_{\mathrm{b}}$ color space. Regarding the transformation between chromaticity values of $\mathrm{RGB}$ on a CRT display and $\mathrm{YC}_{\mathrm{r}} \mathrm{C}_{\mathrm{b}}$, the predecessor has had a long research history $[10,11]$, and the precise transformation relations have been determined as

$$
\left\{\begin{array}{c}
Y=(77 / 256) R+(150 / 256) G+(29 / 256) B, \\
C_{r}=(131 / 256) R-(110 / 256) G-(21 / 256) B+128, \\
C_{b}=-(44 / 256) R-(87 / 256) G+(131 / 256) B+128 .
\end{array}\right.
$$

According to the transformed values between the chromaticity values of RGB on the CRT display and ones of $\mathrm{YC}_{\mathrm{r}} \mathrm{C}_{\mathrm{b}}$, the values of RGB are selected corresponding to the values of the $\mathrm{YC}_{\mathrm{r}} \mathrm{C}_{\mathrm{b}}$ color space that $\pm C_{b}$ changes from \pm 4 to zero in 0.25 intervals in turn when $C_{r}$ is zero and that $\pm C_{r}$ changes from \pm 4 to zero spaced in 0.25 intervals in turn when $C_{b}$ is zero. The mean luminance of the stripes of every grating is maintained at $52.50 \mathrm{~cd} / \mathrm{m}^{2}$ or $54.5 \mathrm{~cd} / \mathrm{m}^{2}$. According to the contrast definition of eq. (2), the contrast values of gratings in the experiment are determined by the values of the $\mathrm{YC}_{\mathrm{r}} \mathrm{C}_{\mathrm{b}}$ color space. Large numbers of pictures with different spatial frequencies and different contrast values are manufactured according to the corresponding values of $\mathrm{R}, \mathrm{G}$ and $\mathrm{B}$ that are selected and combined. The spatial frequencies of pictures are realized by adjusting the width of the stripes. The smallest contrast value of pictures may achieve 0.0092 .

\subsection{Method to determine thresholds}

The testing protocol starts with a dark adaptation time for $30 \mathrm{~min}$ (for each measurement) and then observers view the target gratings on the display screen at a distance of $2 \mathrm{~m}$. Because the display screen may simultaneously demonstrate many gratings, a psychophysical method for multi-contrast interactive thresholds was adopted to determine contrast detection thresholds $[1-3,6]$. The core of the method is to simultaneously display 4 gratings on the display screen with contrast gradually being reduced. The interval of color values $\Delta a^{*}$ (or $\Delta b^{*}$, or $\Delta C_{r}$ or $\Delta C_{b}$ ) of the stripes of four gratings gradually becomes smaller, and the order of the four gratings on each screen is changed arbitrarily. The measurement process is portrayed in Figure 3. The first step is to display four gratings with different and increasing contrasts of $C_{1}, C_{2}, C_{3}$ and $C_{4}$, which gradually become smaller in the beginning of the experiment, and $\Delta a^{*}$ also gradually becomes smaller, which is portrayed in the first map of Figure 3. Observers can determine the most unclear stripe gratings easily, for example, the grating with a contrast value $C_{4}$. The next step is to display four gratings whose contrast and $\Delta a^{*}$ are both lower than that of the contrast of the first step but including $C_{4}$, which is portrayed in the second map in Figure 3. Observers identify the most unclear stripes in the grating again, for example, the grating with the contrast value $C_{7}$. Measurements are then carried out with the same method until observers can barely identify the stripes while

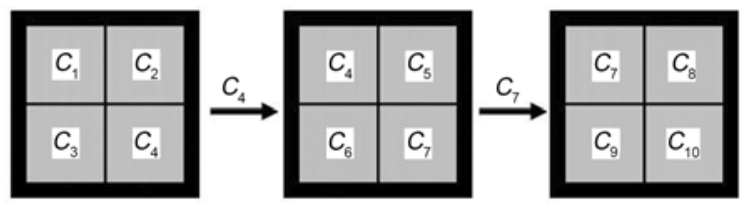

Figure 3 Method for determining contrast detection thresholds.

increasing the contrast gradually and also when reducing the contrast. The contrast values are then the contrast detection thresholds of human vision at that spatial frequency. Measurements are carried out three times for each frequency per person, and all the measurements are repeated once.

\section{Results}

Measurements were carried out on 11 observers using the above method. The relational curve was obtained between mean contrast sensitivity detection thresholds defined in two color spaces and spatial frequencies. The maximum deviation in 11 observers from the average values at each frequency is given in Figure 4.

\section{Discussion}

To date, there have been few studies on measurements of color CSFs with a CRT display. Figure 5 shows the results obtained in 2004 with a monitor by Owens et al. [3] demonstrating that human vision is sensitive to various single colors of red, green, blue and yellow on a color background. The monitor was calibrated with a PR650 chromometer to the $\mathrm{D}_{65}$ standard white, whose CIExy value $x$ was 0.3140 and $y$ was 0.3240 . The observation distance was approximately $1 \mathrm{~m} \mathrm{[3].}$

By analyzing and comparing the experimental results of Owens et al.'s study and this study, it can be seen that they partially resemble each other. The size of the threshold values is the only thing that differs. Thresholds measured by Owens et al. were smaller. The differences may be due to three aspects. The first is that Owens et al. randomly selected a white spot in the Boynton-Macleod color space and took it as the origin of coordinates, and then selected different color directions as color coordinate values. In our study, although opposite color was selected in a similar way, the white spot was selected in the CIELAB and $\mathrm{YC}_{\mathrm{r}} \mathrm{C}_{\mathrm{b}}$ color spaces. Owens et al. did not give a color contrast definition. The second is that the viewing angle was $8^{\circ}-11^{\circ}$ and Owens et al. used six frequencies $(2.53,5.29,9.7,15.54,19.39$ and $29.09 \mathrm{cpd})$. The viewing angle in our experiment was $2.4347^{\circ}$ and the frequencies were $0.4107,0.8215,1.2322$, $1.9714,3.0801,3.7913,4.929,7.041,9.857$ and $16.429 \mathrm{cpd}$. The last is that the color target gratings in our measurements were displayed on a grey background with a luminance of 

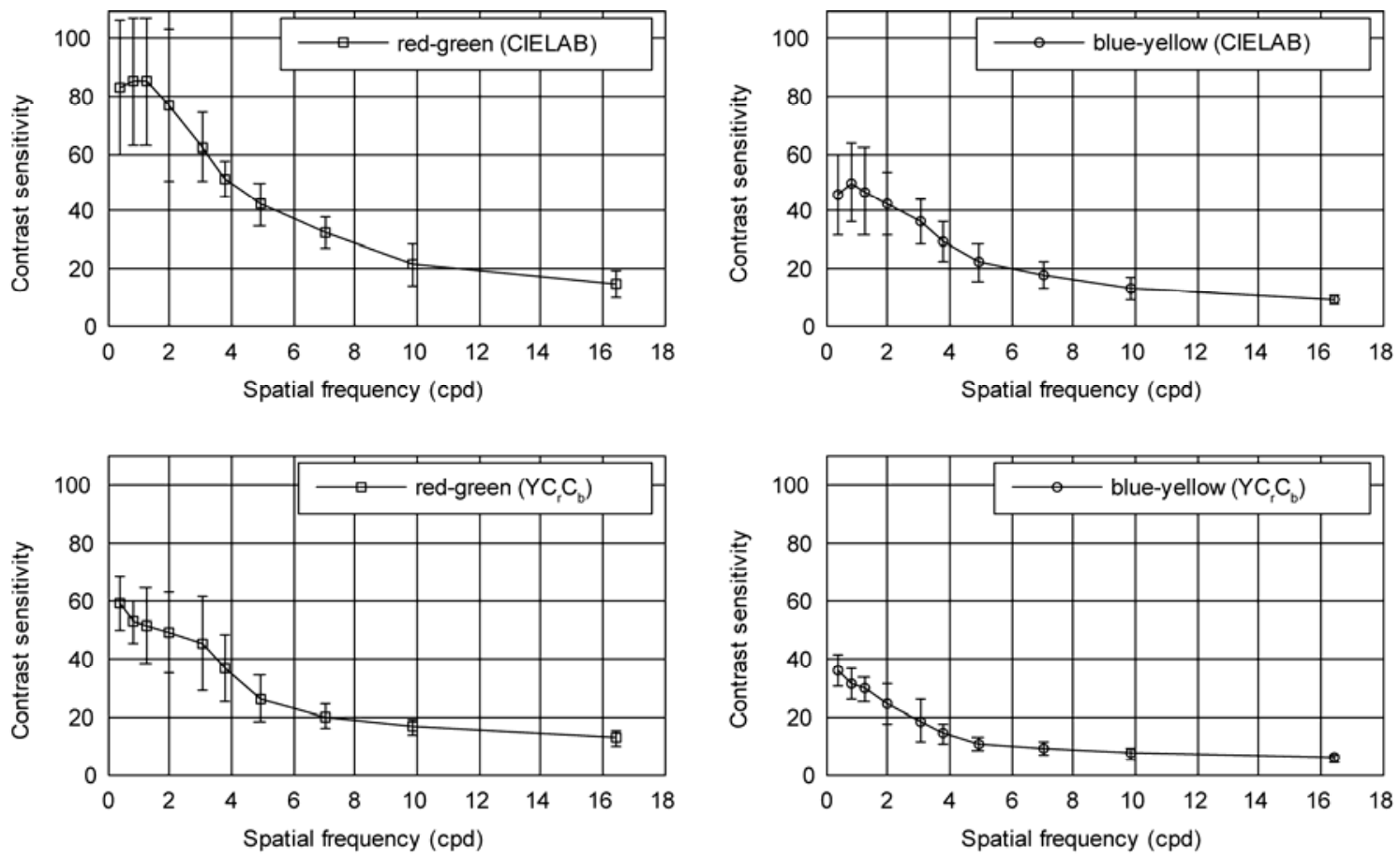

Figure 4 Contrast sensitivity to opposite colors of red-green and yellow-blue measured on a grey background in $\mathrm{CIELAB}_{\mathrm{B}}$ and $\mathrm{YC}_{\mathrm{r}} \mathrm{C}_{\mathrm{b}}$ color spaces.
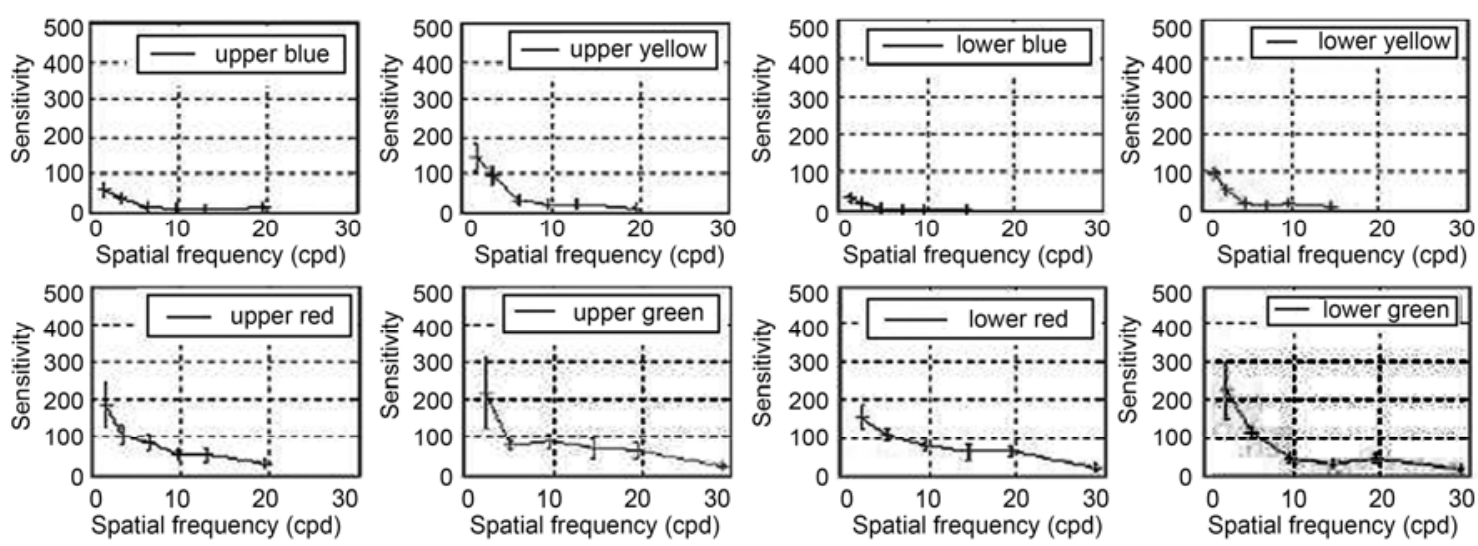

Figure 5 Contrast sensitivity to red-green and yellow-blue measured under weakly chromatic and chromatic backgrounds by Owens et al. [3].

$27.6 \mathrm{~cd} / \mathrm{m}^{2}$, while in the measurements of Owens et al., the gratings were displayed in corresponding weak color or colored backgrounds to study the contrast sensitivity of human vision to various single colors of red, green, blue and yellow [3].

In 2001, Wenzel et al. surveyed the differences in red-green contrast sensitivity using a monitor between normal and defective vision, and the results are portrayed in Figure 6 [5]. Wenzel et al. defined the contrast of red-green color images using the ratio of the spectral radiance energy of the dominant wavelength in the opposite color. Because the goals of their study were mainly to study the differences between normal and defective human vision, the report did not give a description of the measurement conditions and the measurement process in detail. However it may be dis- covered from Figure 6 that the shape of the curves for the results from Wenzel et al. are similar to the results from our study, and the only difference is that the thresholds obtained by Wenzel et al. were generally higher.

When Nadenau [7] studied the image compression algorithm in 2000, he analyzed the contrast sensitivity of human vision to red-green and blue-yellow in CIELAB and $\mathrm{YC}_{\mathrm{r}} \mathrm{C}_{\mathrm{b}}$ color spaces, respectively, on a monitor. Fitted results are portrayed in Figure 7. However, the results are sketchy and the experimental goal was to study the application of a CSF. It was not a study of human vision contrast sensitivity per se. Similarly, measurement conditions and the measurement process in detail are not described.

We performed a non-linear fitting to our data with a least squares method and the results are shown in Figure 8. 


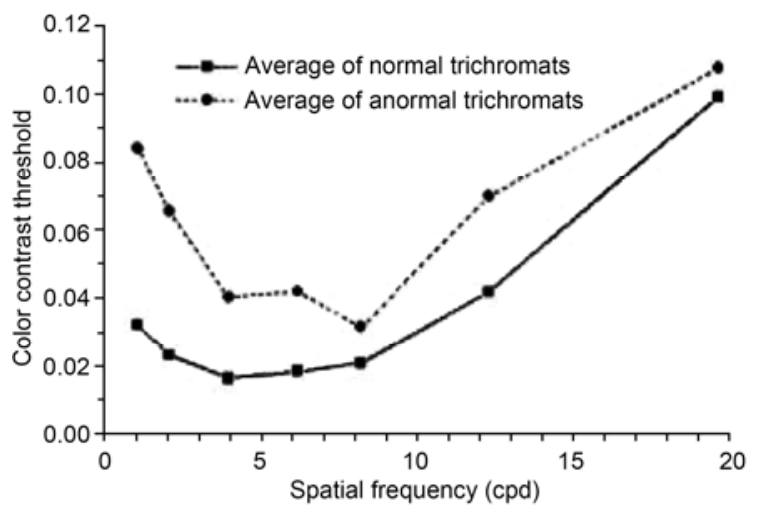

Figure 6 Color CSFs curves of normal and non-normal eyesight measured by Wenzel et al. [6].

Comparing and analyzing our fitted curves with those of Nadenau shows that the shapes of both curves are the same, while the CSFs in our measurements were higher than those from the Nadenau. The second aspect is the mutative tendency of contrast sensitivity values that is basically consistent, but results fitted in Nadenau's measurements changed more quickly. In the $\mathrm{YC}_{\mathrm{r}} \mathrm{C}_{\mathrm{b}}$ color space, when the spatial frequencies were approximately 6 and $14.5 \mathrm{cpd}$, contrast sensitivity values for blue-yellow and red-green colors were smaller than 10. In the CIELAB color space, when the spatial frequencies were approximately 11 and 8 cpd, contrast sensitivity to blue-yellow and red-green colors was smaller than 10. In our study, however, spatial frequencies in the corresponding two color spaces were 13 and 22.5 cpd, respectively, and 15 and $21 \mathrm{cpd}$, respectively, when contrast sensitivity to blue-yellow and red-green color was smaller than 10 .

\section{Conclusions}

Comparing previous and current study results, contrast
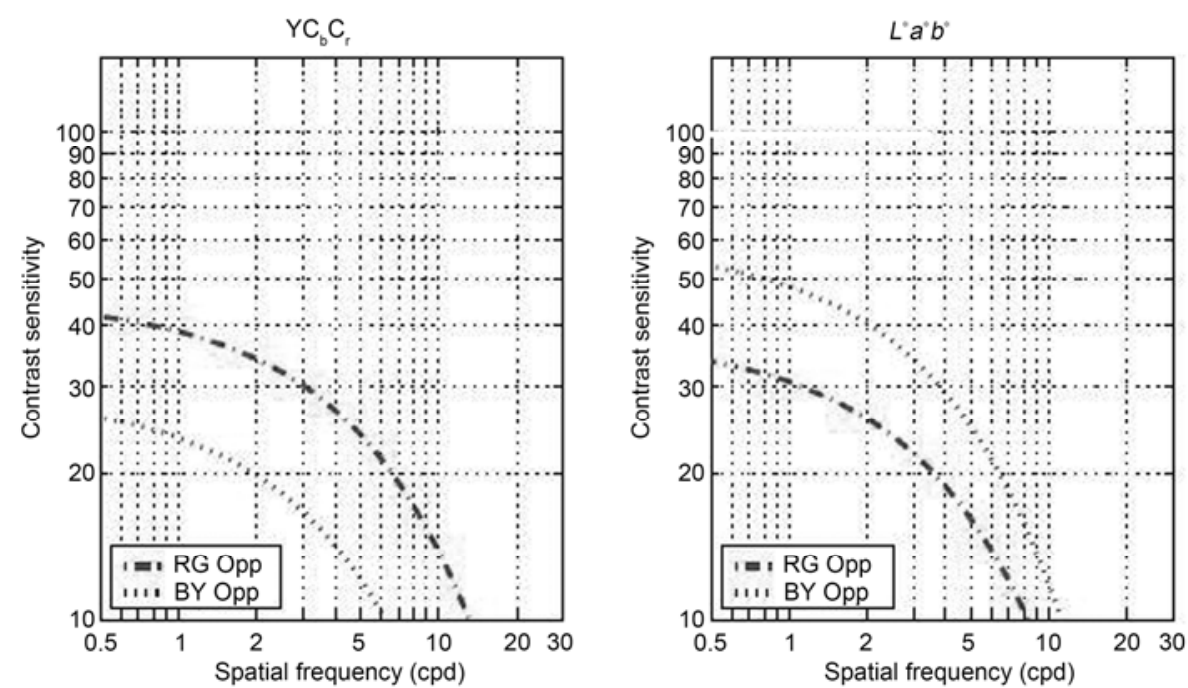

Figure 7 Red-green and yellow-blue CSFs measured by Nadenau [7] in CIELAB and $\mathrm{YC}_{\mathrm{r}} \mathrm{C}_{\mathrm{b}}$ color spaces.
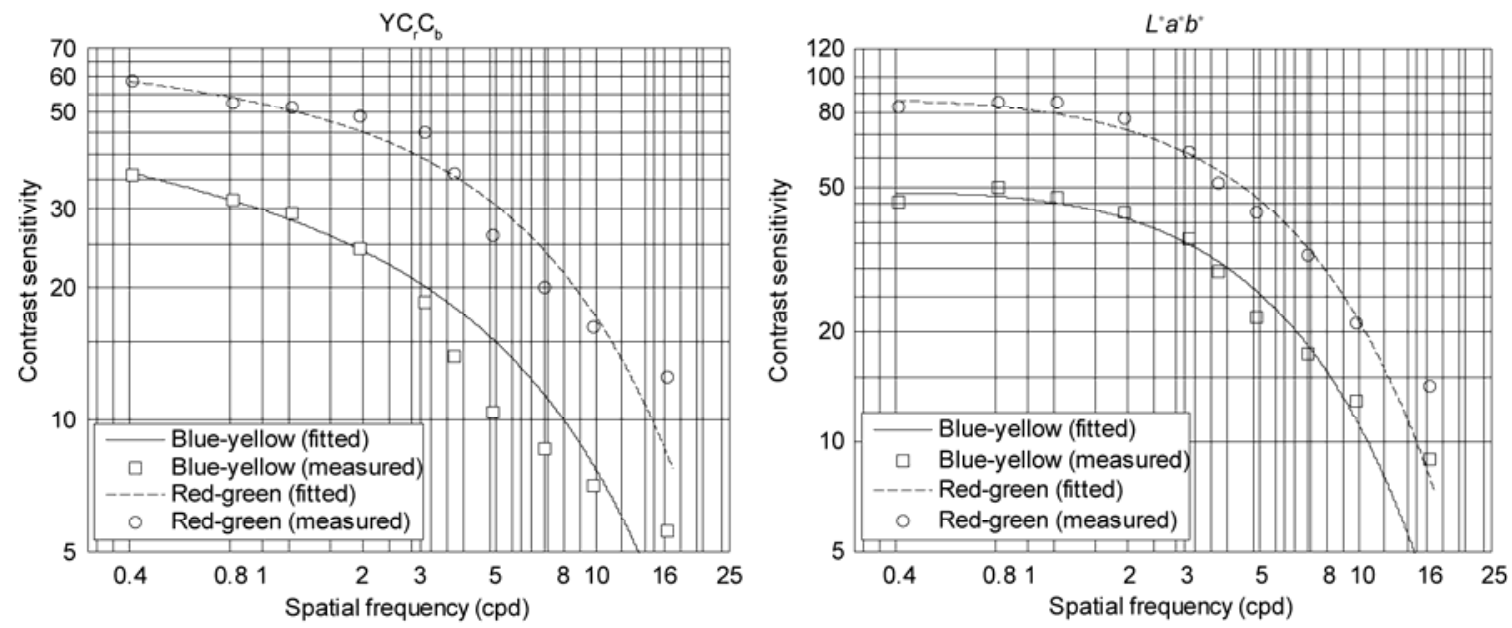

Figure 8 Red-green and yellow-blue opposite color CSFs measured in CIELAB and $\mathrm{YC}_{\mathrm{r}} \mathrm{C}_{\mathrm{b}}$ color spaces with fitted curves. 
detection thresholds were similar but there were some differences. Psychophysical measurements of contrast sensitivity in human vision are influenced by many factors, such as instrument performance, metering equipment precision, experimental environment conditions and experimental techniques.

This work was supported by the National Natural Science Foundation of China (10447005), the Shaanxi Province Science Foundation of China (2009JM1012) and the Scientific Research Plan Project of the Shaanxi Province Education Department of China (2010JK463 and 2010JK464).

1 Petrov Y. Luminance correlations define human sensitivity to contrast resolution in natural images. J Opt Soc Am A, 2005, 22: 587-592

2 Zhang C F, Qiu K, Xu B. Passive optical networks based on optical CDMA: Design and system analysis. Chinese Sci Bull, 2007, 52: 118-126

3 Owens H C, Westland S, Van de V K, et al. Contrast sensitivity for lime-purple and cyan-orange gratings. In: IS \& T/SID Tech Color Imaging Conference, Arizona, USA, 2002. 145-148

4 Valeo E M, Juan L N, Jose A G. Changes in contrast thresholds with mean luminance for chromatic and luminance gratings: A reexamination of the transition from the Devries-Rose to Weber regions. Col Res Appl, 2004, 29: 177-180

5 Wenzel K, Ladunga K, Samu K. Measurement of color defective and normal color vision subjects' color and luminance contrast threshold functions on CRT. Period Polytech Ser Mech Eng, 2001, 45: 103-108

6 Mullen K T. The contrast sensitivity of human color vision to red-green and blue-yellow chromatic gratings. J Physiol, 1985, 359: $381-400$

7 Nadenau M. Integration of human colour vision models into high quality image compression. Ph.D. Thesis. Zurich, Switzerland: Swiss Federal Institute of Technology, 2000

8 Cox M J, Norman J H, Norman P. The effect of surround luminance on measurements of contrast sensitivity. Ophthal Physiol Opt, 1999, 19: 401-414

9 Johnson G M, Fairchild M D. Measuring images: Differences, quality, and appearance. Proc SPIE, 2003, 5007: 51-60

10 Barten P G. Physical model for the contrast sensitivity of the human eye. Proc SPIE, 1992, 1453: 2-15

11 Webster M A. Contrast sensitivity under natural states of adaptation. Proc SPIE, 1999, 3644: 58-70

12 Berns R S. Methods for characterizing CRT displays. Displays, 1996, 16: $173-182$

13 Berns R S. A generic approach to color modeling. Color Res Appl, 1997, 22: 318-332

14 Schütz A C, Delipetkos E, Braun D I. Temporal contrast sensitivity during smooth pursuit eye movements. J Vision, 2007, 7: 1-15

15 Straw A D, Rainsford T, David C, et al. Contrast sensitivity of insect motion detectors to natural images. J Vision, 2008, 8: 1-9

16 Zheng G Y, Du J, Zhang J S, et al. Contrast sensitivity and higher-order aberrations in patients with astigmatism. Chin Med J, 2007, 120: $882-885$

17 Nill N. A visual model weighted cosine transform for image compression and quality assessment. IEEE Trans Commun, 1985, 33: 551-557

18 Elliott S L, Choi S S, Doble N, et al. Role of high-order aberrations in senescent changes in spatial vision. J Vision, 2009, 9: 1-16

19 Bird C M, Henning G B, Wichmann F A. Contrast discrimination with sinusoidal gratings of different spatial frequency. J Opt Soc Am A, 2002, 19: 1267-1273

20 Mullen K T, Kingdom F A. Differential distributions of red-green and blue-yellow cone opponency across the visual field. Visual Neurosci, 2002, 19: 109-118

21 Spehar B, Zaidi Q. Surround effects on the shape of the temporal contrast-sensitivity function. J Opt Soc Am A, 1997, 14: 2517-2528

22 Viqueira V, Illueca C, Ponsa A M, et al. Influence of the luminance spatial non-homogeneities of a display monitor on the contrast sensitivity function determination. Displays, 1999, 20: 65-71

23 Vidan G A, Harel M, Hendler T. Contrast sensitivity in human visual areas and its relationship to object recognition. J Neurophys, 2002, 87: 3102-3116

24 Peli E. Contrast sensitivity function and image discrimination. J Opt Soc Am A, 2001, 18: 283-293

Open Access This article is distributed under the terms of the Creative Commons Attribution License which permits any use, distribution, and reproduction in any medium, provided the original author(s) and source are credited. 